\title{
Inserción laboral de jóvenes con discapacidad. Análisis de las prácticas laborales ${ }^{1}$
}

\author{
Martina Pérez Serrano \\ UNIVERSIDAd AUTÓNOMA DE MADRID
}

\section{Resumen}

Esta investigación se centra en la inserción laboral de los jóvenes con discapacidad intelectual. Su objetivo consiste en analizar el periodo de prácticas laborales recogiendo la voz de los protagonistas. Se ha utilizado la metodología cualitativa para el análisis de los cuadernos de prácticas de alumnos y diarios de prácticas de las profesoras tutoras. El tratamiento de los textos en sus dos niveles -textual y conceptual- se ha llevado a cabo con el programa informático Atlas.ti 5.2. Los resultados del estudio han puesto de relieve que es posible la inserción laboral de las personas con discapacidad. Se precisa de la colaboración de todos los implicados -Universidad y Empresa receptora de alumnos en prácticas-. Así como de un diseño de formación práctica adecuada a las peculiaridades de los sujetos y demandas del mundo laboral.

Palabras Clave: Discapacidad intelectual, Jóvenes, Investigación cualitativa, Inserción laboral, Inclusión social.

\section{Summary}

This research is focused on the work inclusion of intellectual handicapped youths. Its aim is to study the period of work practices by including the protagonists' voice. A qualitative methodology for the practice diaries (pupils and teacher tutors) has been used. The text processing at both levels-textual and conceptual- has been developed by means of the computer application Atlas.Ti 5.2. The results of our study have featured that the work insertion of handicapped people is possible. To achieve this goal, we need the collaboration of all the involved agents: university, business world, as well as a practical formation that is tailored to the peculiarities of individuals and the demands of the labour force.

KEY WORDS: Intellectual handicap, Youths, Qualitative research, Work inclusion, Social inclusion.

$\square$ l trabajo forma parte de una investigación más amplia sobre la formación de jóvenes con discapacidad intelectual para la inserción laboral. Tiene por finalidad dar respuesta a las demandas de formación orientada al empleo. El programa se desarrolla durante dos cursos académicos en el marco de la Universidad Autónoma de Madrid. Al finalizar este periodo, los jóve- 
nes se incorporan a un puesto de trabajo adecuado al perfil de cada uno de ellos. Comprende tres ámbitos: periodo de formación, inclusión en el entorno universitario e inserción en el mundo laboral. Este último constituye el objetivo del presente estudio. Las prácticas laborales forman parte de la organización de las enseñanzas curriculares seguidas por los alumnos con discapacidad intelectual. Persigue que los alumnos alcancen las competencias laborales, personales, relacionales y manipulativas pertinentes para el desempeño de un puesto de trabajo acorde con sus capacidades y cualificaciones.

Uno de los desafíos de la sociedad actual es el de enfrentarse al reconocimiento y la valoración de la diversidad humana, tratando de superar los modelos de organización normalizados en los que las personas con discapacidad veían disminuidas sus oportunidades de participación. En la actualidad se formulan políticas destinadas a promover la plena participación e intervención de las personas con discapacidad en los procesos económicos, sociales y educativos inspirados en el principio de igualdad de oportunidades para todos los ciudadanos. Pues como señala Ríos (2003, 294), "todo ser humano tiene derecho a aprender".

Es evidente que el ámbito laboral desempeña un papel primordial en la inclusión social de estos sujetos. Por lo general, la integración laboral implica que los empleados con y sin minusvalía estén integrados en iguales condiciones dentro de la misma red social en el lugar de trabajo.

Este artículo se destina a presentar la investigación realizada sobre la implementación y desarrollo del periodo de prácticas. Sobre este eje central pivotan nuestras preocupaciones. Persigue como objetivo descubrir cómo se ha llevado a cabo el periodo de prácticas laborales a través de las narraciones que los protagonistas han escrito en los cuadernos de experiencias (los alumnos) y en los diarios de prácticas (profesoras tutoras). En dichos cuadernos han plasmado de forma secuenciada las diferentes situaciones vividas.

\section{Metodología cualitativa. Investigación con diarios}

Este trabajo se centra en el análisis de los diarios-cuadernos de experiencias, constituyendo estos la principal herramienta de recogida de información. Se persigue penetrar y descubrir a través de sus narraciones, sus vivencias, sentimientos y las relaciones experimentadas. Se ha recogido también una valiosa información a través de los diarios de prácticas de las profesoras tutoras. Este estudio nos permite captar aquello que subyace en los escritos con el fin de descubrir los puntos diana.

Se ha trabajado con un grupo de 16 jóvenes (18 a 30 años) con discapacidad intelectual que realizan el periodo de prácticas. Y tres profesoras tutoras.

Para llevar a cabo este trabajo nos hemos apoyado en la grounded theory (teoría fundamentada). Esta teoría se descubre, se desarrolla y se verifica provisionalmente a través de la recolección y análisis sistemático de datos sobre el fenómeno estudiado. Desde esta óptica, la teoría es el resultado de la conceptualización basada en la interpretación de datos, que provienen de una realidad sobre la que no existía una modelización previa. No basta la simple descripción de la realidad y de los hechos, sino que hay que penetrar en las redes del significado para llegar a comprender lo que realmente sucede y por qué sucede.

El procedimiento seguido en el trabajo ha consistido en los siguientes pasos:

1. Recogida de información en contextos naturales en los cuales realizaban las prácticas laborales por medio del cuaderno de prácticas (alumnos) y el diario de prácticas (tutoras). En ellos, los autores volcaron 
sus reflexiones, observaciones y preocupaciones diarias. Dichos instrumentos constituyen las herramientas a utilizar en todo el proceso de investigación.

2. Planteamiento del problema y formulación de objetivos e hipótesis de trabajo. 3. Descripción de la muestra.

4. Análisis de los datos. Determinación de las unidades de análisis.

5. Posteriormente y mediante la comparación constante de los datos realizamos la interpretación de los mismos a fin de obtener conclusiones y aportaciones para la validación del programa formativo de los alumnos con discapacidad.

El problema planteado consiste en analizar en qué medida el periodo de prácticas ha contribuido a la formación laboral de los alumnos. Los objetivos del estudio y las hipótesis de trabajo han emergido de las lecturas pausadas de los textos, de sus contenidos y sus interrelaciones. Concretamente, los interrogantes son los siguientes: ¿cuáles son los ángulos de interés que reflejan los alumnos y las profesoras tutoras? ¿qué temáticas específicas suelen destacar? ¿desde qué perspectivas? ¿qué competencias consideran los protagonistas que están adquiriendo en el periodo de prácticas? ¿cuál es el clima socio-relacional que se establece entre los alumnos y los compañeros en los lugares de trabajo? ¿qué funciones desempeñan las profesoras tutoras? ¿qué repercusiones ha tenido en los implicados la participación directa en el periodo de prácticas? ¿cómo valoran dicho periodo?

Las hipótesis de trabajo que guían nuestras reflexiones son las siguientes:

1. Si el programa de prácticas laborales es adecuado para preparar al alumnado para el buen desempeño del puesto de trabajo. 2. Si las funciones desempeñadas por las profesoras tutoras de prácticas son las pertinentes.

3. Verificar si el mediador laboral es un recurso imprescindible para la inserción la- boral de los alumnos con discapacidad. 4. Constatar cómo valoran los implicados (profesores tutores, mediadores y empleadores) el periodo de prácticas laborales.

En suma, se trata de estudiar el periodo de prácticas desde la perspectiva de sus protagonistas: los alumnos, los responsables de la empresa y las profesoras tutoras.

\section{Análisis de los datos. Programa Atlas.ti}

Para el análisis e interpretación de los datos textuales hemos utilizado el programa informático Atlas.ti 5.2, que nos ha ayudado en el manejo de los textos, facilitando su organización, búsqueda y recuperación. Se incluye dentro de los CAQDAS de última generación, constructores de teorías. El proceso de análisis lo desarrolla en dos fases (textual y conceptual) estrechamente relacionadas e interconectadas, como un continuo ir y venir entre ambas, dando lugar a un proceso recursivo y retroalimentador entre ellas. Ambos tipos de análisis dan lugar al establecimiento de relaciones entre elementos, a la construcción de redes y a la representación gráfica facilitando la interpretación. El programa Atlas.ti ayuda a descubrir los fenómenos complejos que se encuentran ocultos en los datos cualitativos.

Los pasos a seguir en la utilización de este programa son los siguientes: a) Creación de la unidad hermenéutica. b) Asignación de los documentos. c) Herramientas textuales: marcado y codificación. d) Herramientas conceptuales. Códigos. e) Memos: comentarios. f) Familias: conjunto de objetos que comparten alguna cualidad. g) Redes (network) que conforman una red de relaciones. h) Generación de informes.

En el trabajo se ha procedido del siguiente modo:

- Preparación de los documentos textuales: recogida y asignación de los textos en la "unidad hermenéutica". 
- Realización del análisis inicial. Exploración de las ideas centrales mediante lecturas selectivas, segmentación del texto, creación de citas textuales, elaboración de códigos y anotaciones explicativas.

- Análisis principal. Realización del análisis conceptual. Creación de familias de códigos nuevos y de relaciones.

- Resultados e interpretación. Construcción de las redes de conceptos significativos. Exposición de los resultados encontrados de forma intuitiva mediante gráficos y redes conceptuales.

- Conclusiones del trabajo.

La codificación es la fase de asignar a cada unidad segmentada -cita-, un distintivo, un código. Estos códigos son provisionales, tienen una función comparativa y se fundamentan en los datos. Este primer nivel de codificación lo denominamos inicial. Por su carácter abierto facilitan nuevas posibilidades de análisis dando lugar a un segundo nivel de codificación. Este segundo nivel de codificación consiste en realizar separaciones de códigos iniciales organizándolos por clases de forma que incluyan los pertenecientes a cada una de las diversas clases o categorías. Para llevar a cabo este segundo nivel de codificación se procedió del siguiente modo: a) Una lectura comprensiva de cada código inicial junto con las citas asignadas realizando comparaciones entre ellos. b) Más tarde se organizaron los primeros códigos en clases y las nombramos con conceptos-ideas más generales con el fin de buscar respuestas a los interrogantes planteados en el estudio. Se denominan códigos genéricos. Para obtener estos conceptos más genéricos se acudió de nuevo a los textos originales, a los vocablos empleados por los protagonistas, y desde ahí emergieron las denominaciones de los conceptos nuevos tratando de recoger globalmente el sentido de lo que hacen, cuentan, sugieren y expresan. Procuramos recabar de sus acciones y actitudes lo que nos quieren comunicar, solicitar y sugerir. Este momento del proceso de análisis y codificación de los datos nos conduce al tercer nivel de codificación el cual arranca desde el descubrimiento de los temas conceptuales en los datos, esto es, desde los códigos genéricos anteriores obtenidos en el segundo nivel de codificación. Partiendo de ellos se realizaron nuevas comparaciones de los enunciados entre sí con el fin de indagar si existe un concepto más amplio capaz de unificarlos. Con la identificación hallada de los elementos comunes y diferenciadores nos ha permitido configurar un esquema de categorías o ejes organizadores en torno a los cuales han quedado integrados y relacionados los códigos genéricos. Este nivel de codificación algunos autores lo denominan codificación axial y categorización otros. El empleo y manejo de estos tres niveles de codificación nos ha permitido la construcción del listado de categorias o ejes organizadores en el estudio.

El listado de categorías o ejes organizadores obtenidos en el tercer nivel de codificación es el siguiente: a) Diseño organizativo del periodo de prácticas laborales. Este eje agrupa y relaciona varios códigos genéricos referidos a la planificación del periodo; tareas para introducir al alumno en el puesto de trabajo y organización del seguimiento del alumno. b) Aspectos relacionales durante el periodo de prácticas. Este eje agrupa los siguientes códigos genéricos: actitudes personales ante el trabajo; cómo se muestran; comportamientos y relaciones. c) Cooperación de los responsables durante el periodo de prácticas. Este eje reúne los códigos relativos a los roles y funciones que han desempeñado los responsables de los escenarios de prácticas. Los códigos genéricos que incluye se refieren a los responsables cuando participan, se muestran y se implican. d) Funciones de las profesoras tutoras. En él se han incluido temáticas específicas relativas a los roles y funciones. 
Tareas tales como asesorar, realizar el seguimiento, evaluar, visitar a los alumnos en los puestos de trabajo, así como sus expectativas de futuro.

En las redes se presenta una visión global de los datos, a partir de ellas se pueden visualizar las relaciones entre los códigos y las familias de códigos. Para la creación de las familias de códigos hemos seguido criterios de pertenencia, de proximidad e inclusión y de concurrencia.

\section{Interpretación de los datos}

La interpretación de los datos en el análisis cualitativo representa la fase creativa en la que el investigador debe conjugar armónicamente los resultados obtenidos en el estudio con el marco teórico subyacente. La interpretación de los datos ocupa un lugar importante. Consiste en guiar y explicar los resultados a la luz de una teoría adecuada al objeto de estudio. Al presentar los resultados pretendemos no solo la descripción, sino también su comprensión, e integrar todo el trabajo en un marco teórico más amplio que ilumine los interrogantes e hipótesis.

La interpretación de los resultados exige una reflexión sistemática y crítica de todo el proceso con el fin de contrastar los resultados con la teoría. Esto es, verificar la adecuación entre el objeto y el pensamiento. Es la fase más importante porque implica la toma de decisiones sobre el modo de transformar y cambiar el modelo de prácticas laborales. Para realizar este proceso con rigor hemos avanzado siguiendo estas fases: primero, a través de la comparación de los datos textuales se definieron los códigos iniciales y los genéricos, segundo, se establecieron nuevas relaciones entre los códigos genéricos, tercero, con el fin de encontrar nuevos conceptos que los aglutinen emergieron las categorías o ejes organizadores. La presentación de los datos se lleva a cabo mediante redes que muestran los ejes organizadores, que a su vez integran los códigos genéricos obtenidos en el segundo nivel de codificación.

\section{a) Diseño del periodo de prácticas laborales}

La red denominada diseño del periodo de prácticas laborales agrupa los siguientes códigos genéricos: planificación del periodo; plan de tareas para introducir al alumno; plan de seguimiento y organización del seguimiento. La relación establecida entre ellos expresa en qué medida los diferentes códigos genéricos facilitan el diseño, el desarrollo y organización del periodo de prácticas. Todos ellos interactúan armónicamente contribuyendo a la calidad.

La labor de orientación y seguimiento las profesoras tutoras la han organizado mediante reuniones al inicio, durante y al final de la jornada. En las reuniones bien de inicio o de final de jornada recuerdan a los alumnos cómo se va a realizar el seguimiento. Lo expresa así: "el seguimiento se realizará mediante tres vías: ellos tendrán que entregarme un cuaderno de experiencias. Algunos alumnos contarán con voluntarios, mediadores laborales que les ayudaran a su adaptación al trabajo para que les resulte más fácil. Por último, cada dos semanas realizaremos charlas en las que recogeremos sus inquietudes, sensaciones o problemas, y trataremos de reforzar actitudes y competencias".

Es importante no descuidar ninguno de los elementos que han puesto de relieve los protagonistas para la adquisición de las competencias. Conviene subrayar que la buena planificación del periodo y su seguimiento ha contribuido a que los alumnos se sientan guiados, sabiendo lo que tienen que hacer y cómo hacerlo. En sujetos de estas características han jugado un papel significativo la aclaración de normas y la reiteración de las mismas. Lo que les ha permitido insertarse en los escenarios de prácticas laborales. 


\section{b) Aspectos relacionales durante el periodo de prácticas laborales}

Se trata de una red de síntesis que agrupa los códigos genéricos que se visualizan en la red en torno al supercódigo o código globalizador, denominado aspectos relacionales. Entre todos los códigos genéricos se han establecido relaciones. Unas ponen de relieve cómo determinados códigos genéricos facilitan o están asociados a los aspectos relacionales en el trabajo entre los alumnos y los compañeros, y otras relaciones, sin embargo, reflejan aquello que las impiden y dificultan. Podemos destacar la carencia de habilidades sociales en algunos casos, como la mentira, la pasividad, no mirar a la cara, ni saludar a los compañeros. Como aspectos positivos, la pun- tualidad, el agradecimiento, el sentido de responsabilidad, el querer ser eficaces... "Tiene un comportamiento totalmente adecuado, correcto e impecable. Su actitud es buena". Los alumnos en el trabajo muestran actitudes positivas, como por ejemplo "estoy satisfecho, contento, ilusionado, triste, sorprendido". Otros, sin embargo, narran: "me sentía asustada por la cantidad de competencias que tenia que realizar".

\section{c) Cooperación de los responsables durante el periodo de prácticas}

Este eje organizador se entiende como uno de los puntos fuertes del periodo de prácticas laborales, pues los responsables desarrollan en todo el periodo una gran labor

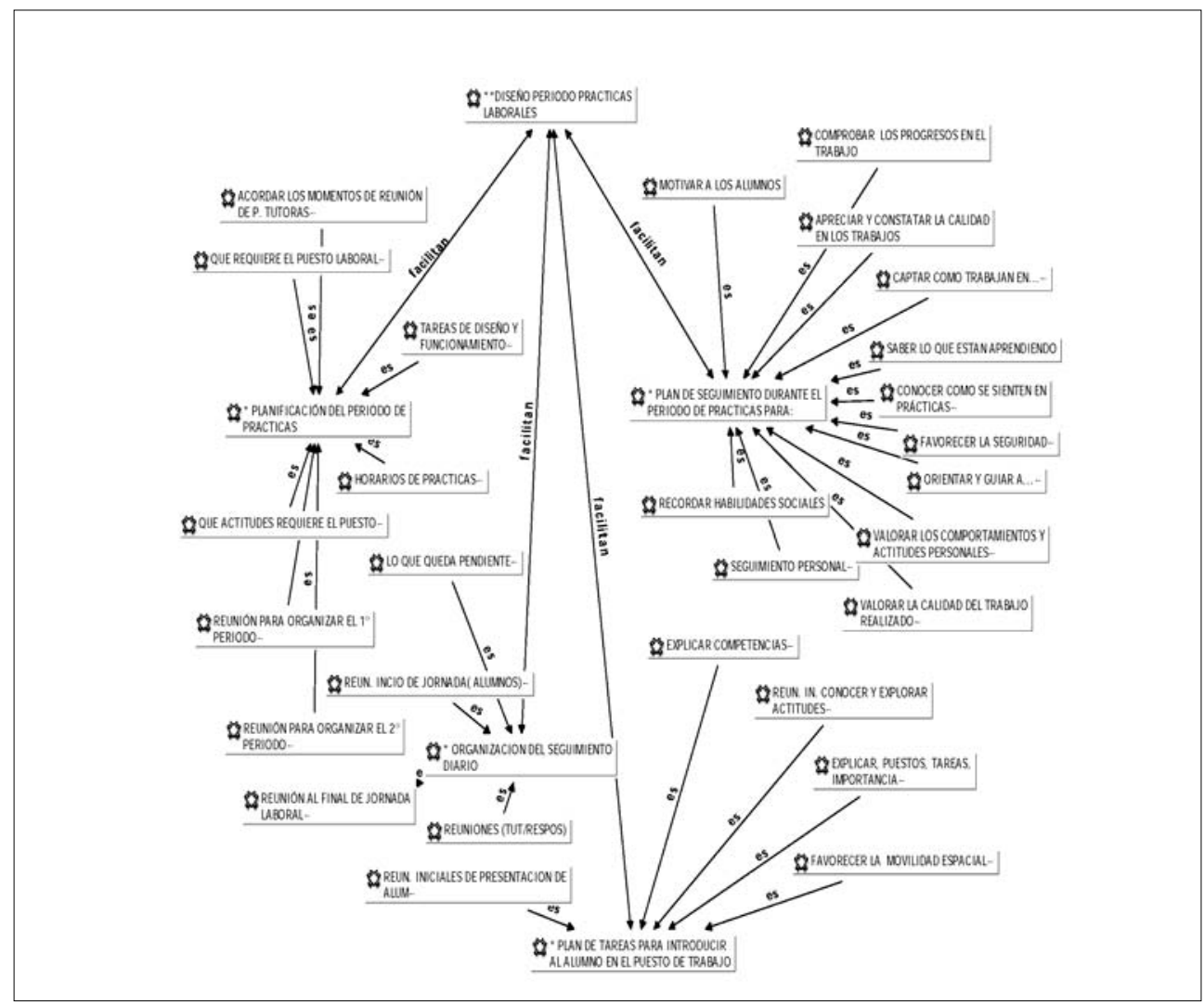

Diseño del periodo de prácticas laborales. 


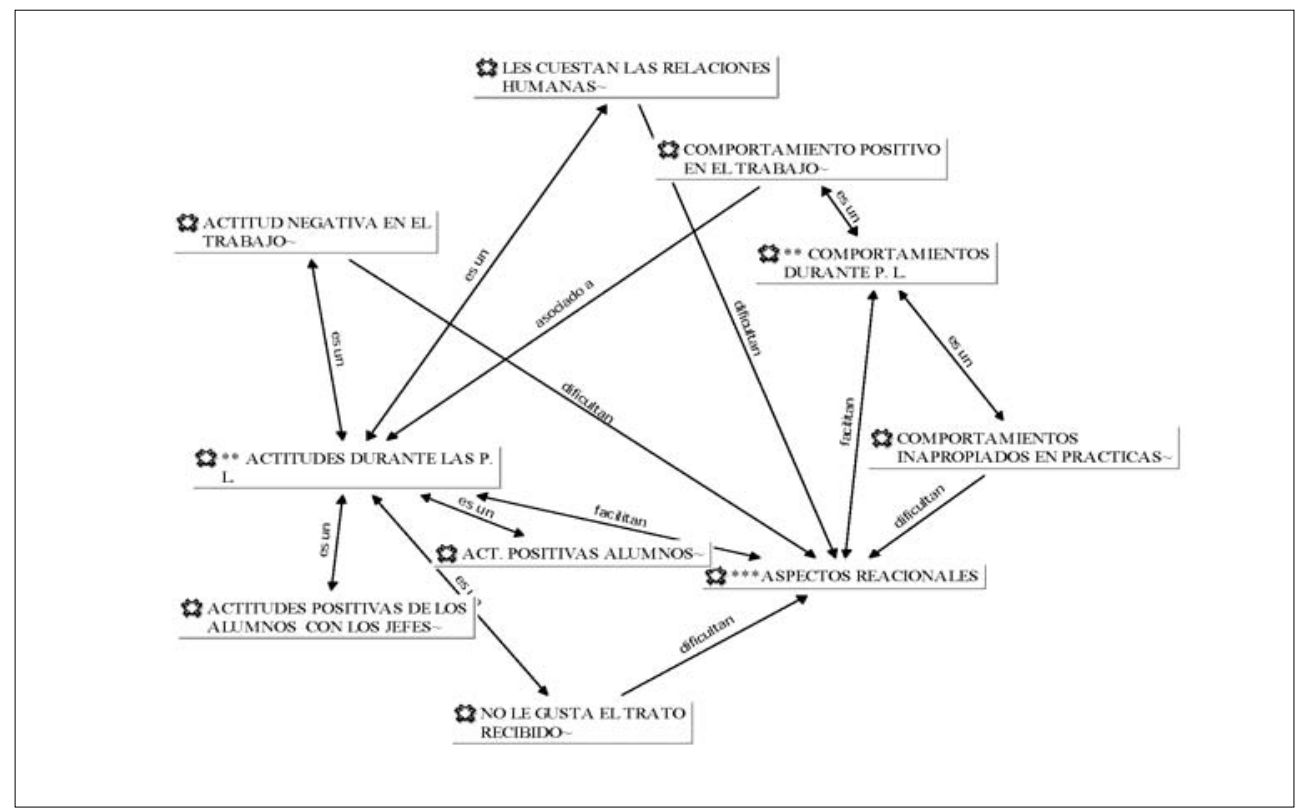

Aspectos relacionales durante el periodo de prácticas laborales.

formativa por su enorme participación, implicación y actuación. Han participado activamente en proponer las tareas que los alumnos deben realizar, en explicar y comprobar cómo las realizan. Las profesoras tutoras mantienen contactos frecuentes con los responsables acerca de la evolución y aprendizajes adquiridos por los alumnos. La siguiente cita tomada de sus diarios lo expresa: "ha trabajado despacio pero lo que hace, lo hace correctamente". La implicación de los responsables hace referencia a los aspectos de observación, orientación y seguimiento que realizan de los alumnos en el trabajo, en concreto como lo hacen; resuelven las dudas, les demuestran cómo se hace paso a paso. Los responsables exponen a las profesoras tutoras sus preocupaciones acerca de la formación que deben dar a los alumnos. Por ejemplo una de las profesoras tutoras ha narrado en su diario: «La jefa me expone "estoy preocupada porque no se si los trabajos propuestos son adecuados"»; "Tengo miedo de perjudicar al alumno, en vez de beneficiarle".
La siguiente red, denominada cooperación de los responsables durante el periodo de prácticas, representa las relaciones establecidas entre todos los códigos genéricos referidos a los responsables que participan, se implican y se muestran, comentados anteriormente. Con ellos hemos construido un supercódigo o código globalizador denominado cooperación de los responsables $\mathrm{du}$ rante el periodo de prácticas. En la red se capta con claridad su participación en la labor formativa, así como sus preocupaciones educativas y didácticas. Desconocen cómo dirigirse a los alumnos y explicarles las tareas a realizar. Se preguntan si su comportamiento es el adecuado. Con frecuencia se muestran permisivos, tolerantes y poco exigentes. En general muestran buena disposición y colaboración. Los responsables de los diferentes puestos de trabajo han desempañado un papel imprescindible en el periodo de formación en prácticas laborales puesto que sin su implicación directa no habría sido posible llevar a cabo la inserción laboral de estos jóvenes. 


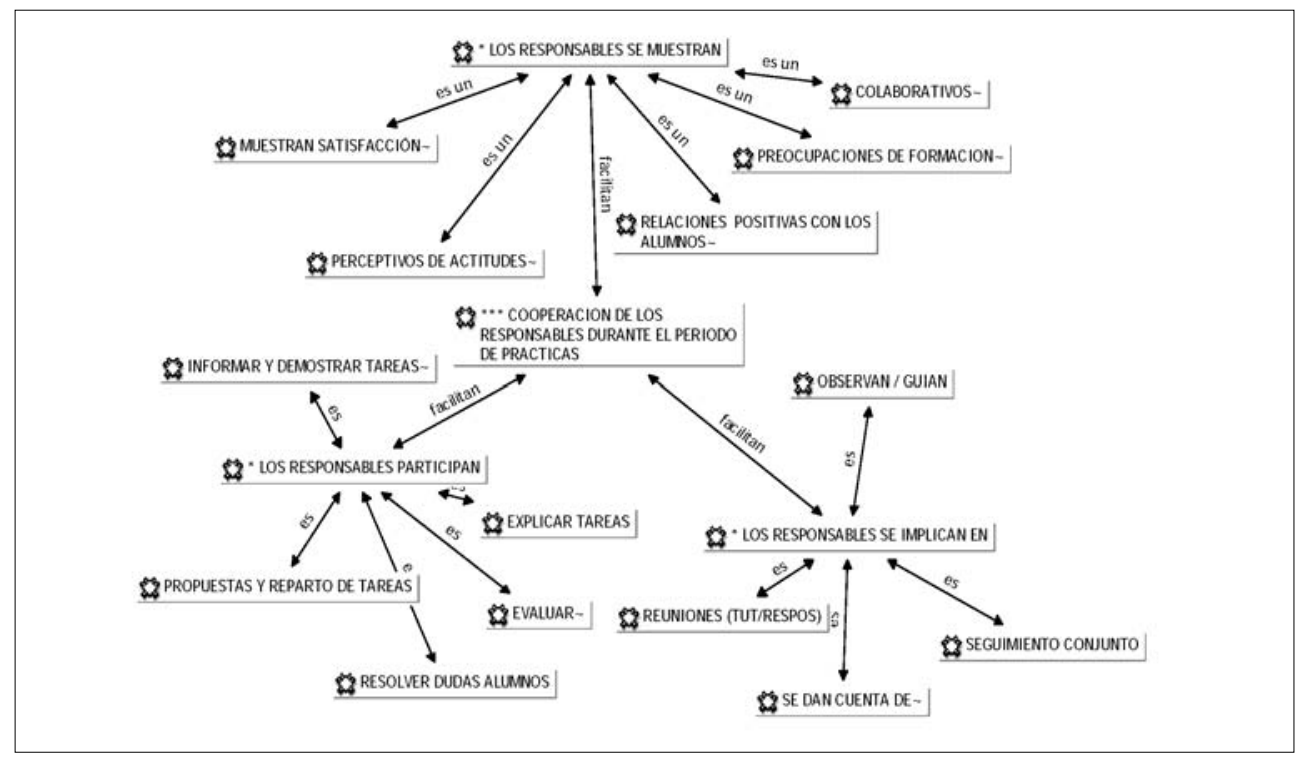

Cooperación de los responsables durante el periodo de prácticas.

\section{d) El papel de las profesoras tutoras}

El papel de las profesoras tutoras se deja traslucir en todas las redes anteriores dada su transversalidad. Muchas son las funciones que han desempeñado a lo largo de todo el periodo. Las cuales se resumen a continuación.

Un papel esencial de las profesoras tutoras es el de orientar y asesorar a los responsables en la acción metodológica a la hora de explicar y exigir las tareas. La forma de abordar esta orientación y asesoría ha sido por medio de conversaciones. La profesora tutora conversa con los responsables acerca de los posibles abordajes metodológicos con el fin de que en la explicación de la tarea "ordenen secuencialmente los pasos a seguir en la ejecución de la misma para que los alumnos no se confundan en su realización". En relación con el nivel de exigencia, las profesoras tutoras les orientan del siguiente modo: "No tengáis problemas en exigirles y corregirles, esto no les perjudica, sino todo lo contrario". Ante la pregunta “'hasta dónde debo exigirle al alumno?". Las tutoras responden: "podéis ir solicitándoles actividades cada vez más complejas, sólo la respuesta del alumno en el rendimiento os irá marcando el nivel de exigencia”.

Otro de los papeles desempeñados a lo largo del periodo se centra en favorecer la estimulación y motivación de los alumnos en cooperación con los responsables. En esta cita se capta este rol. La profesora tutora recoge en su diario la conversación mantenida con el jefe responsable del puesto de trabajo. Le comenta: "A. debe superar su timidez, ya que ella tiene capacidades que pueden verse mermadas por su timidez". Afirma que en "el momento en el que se sienta cómoda y relajada, va a realizar un trabajo mucho más rápido y fluido, estoy convencida de que va a hacerlo bien".

Las profesoras tutoras realizan con frecuencia valoraciones de los puestos de trabajo, así como de los responsables empresariales por medio de visitas a los escenarios de prácticas y conversaciones mantenidas con ellos. Las valoraciones por lo general son positivas: destacan lo orgullosos que están del trato dispensado a los alumnos. Estos comentan que han estado muy a gusto trabajando con ellos. 
La profesora tutora, por su parte, señala la enorme paciencia que han tenido y su implicación e interés con el programa de formación laboral. En cuanto a la valoración de los espacios laborales, consideran que todos son los adecuados para estos sujetos con discapacidad intelectual.

Rol de autoevaluación. Las profesoras tutoras evalúan todos los aspectos del plan de prácticas laborales y su labor de seguimiento de los alumnos tutelados. En sus diarios recogen: "el seguimiento realizado durante el periodo de prácticas es positivo porque a los alumnos les ha ido fenomenal; les ha servido para recordar los pasos a seguir sin tener que preguntar cómo hacer una u otra tarea; nos parece una buena experiencia para el resto de los alumno/as el pedirles este cuaderno en años sucesivos; será útil para obtener información y para diseñar mejor sus perfiles laborales".

\section{Conclusiones del estudio y propuestas de acción}

Las conclusiones son la quintaesencia de la investigación. Ofrecen la posibilidad de aportar claves que iluminen la búsqueda de respuestas.

La investigación realizada permite obtener explicaciones rigurosas sobre diferentes elementos a tener en cuenta para el buen funcionamiento del periodo de prácticas laborales. Despierta en los protagonistas nuevas expectativas, a la vez que se sienten partícipes implicándose en todo el proceso. Supone un contacto directo con la realidad, lo que nos permite detectar los problemas, dificultades y limitaciones subyacentes. Favorece el trabajo cooperativo entre los profesores tutores, los responsables de la empresa y los alumnos aportando una visión multidisciplinar

Los protagonistas nos han manifestado sus deseos, necesidades, intereses, senti- mientos, así como el nivel de comportamiento en los puestos laborales. En este estudio se ha intentado responder a las preguntas e hipótesis formuladas. Las conclusiones se han estructurado en torno al plan de prácticas y los diferentes elementos intervinientes en el mismo: las profesoras tutoras, los mediadores laborales, la familia, los alumnos y los responsables de la empresa.

El periodo de prácticas laborales tiene por misión prioritaria insertar a estos jóvenes en la vida activa. Se pretende que lleguen a alcanzar el máximo nivel de independencia posible en función de sus capacidades en los diferentes entornos. Este periodo ha contribuido a que los alumnos puedan realizar tareas laborales que les propicien la inserción laboral en el mercado ordinario de trabajo.

Conviene reseñar que el periodo de prácticas ha incrementado su autoconcepto de forma significativa al experimentar que son capaces de realizar determinadas tareas por sí solos. Este incremento favorece, sin duda, el nivel de aspiraciones y posibilidades.

Tanto los alumnos como los responsables de la empresa y las profesoras tutoras consideran que el diseño del plan de prácticas es adecuado, si bien precisa de algunos reajustes, fundamentalmente referidos a los responsables de la empresa y en lo que afecta a las relaciones sociales con los alumnos de discapacidad intelectual. Todos los elementos organizativos que se han tenido en cuenta para el diseño y desarrollo del periodo han constituido un entramado armónico que ha contribuido al buen funcionamiento del mismo propiciando la adquisición de las competencias previstas en cada puesto de trabajo. Es de ensalzar la alta implicación de los responsables de los puestos laborales en los diferentes ámbitos formativos, preocupándose tanto de desarrollo de habilidades manipulativas como sociales y cognitivas.

El papel desempeñado por las profesoras tutoras es crucial en todo el desarrollo 
del periodo de prácticas laborales. Podría afirmarse que constituyen el eje vertebrador del periodo de prácticas tanto en lo referido al diseño, organización e implementación como a la hora de realizar el seguimiento personalizado de cada alumno en los puestos laborales: constituyen el punto de referencia para los alumnos, los mediadores y los responsables de la empresa en los puestos de trabajo. Entre las principales funciones realizadas, subrayamos:

- Diseñar, organizar y evaluar el periodo de prácticas.

- Orientar a los responsables laborales acerca de cómo deben tratar y relacionarse con los alumnos.

- Asesorar en la metodología didáctica con el fin de facilitar la comunicación entre los responsables laborales y los alumnos.

- Estimular el desarrollo de habilidades sociales.

- Apoyar a los alumnos en el desarrollo de las prácticas laborales resolviendo los problemas cotidianos.

- Realizar el seguimiento diario de los alumnos tutelados.

- Evaluar a los implicados.

- Autoevaluación de las funciones realizadas y sugerencias de mejora.

El comportamiento de los alumnos en prácticas ha sido el adecuado a distintos niveles y se ha visto mejorado a lo largo de todas las prácticas. Las manifestaciones conductuales que se han observado van en la línea de: la satisfacción y el contento han sido manifiestos en todos los alumnos, si bien en ocasiones algunos han expresado cierto grado de ansiedad al no saber resolver la situación que se les presentaba. Son puntuales, constantes, trabajadores y con ganas de aprender. Muchos de ellos muestran iniciativa y gran soltura en el trabajo. Las tareas que más les gustan son las que se realizan con el ordenador, las de secretaría, biblioteca: colocar libros, tejuelar, de carácter mecánico y rutinario. En este aspecto son eficaces puesto que no quieren descansar hasta no finalizar la tarea. En general acatan muy bien las normas de los jefes laborales y acuden a ellos siempre que lo precisan. A través de las relaciones establecidas con los jefes y otro personal en los espacios de prácticas se les han ampliado los horizontes considerablemente. Valoran positivamente los diferentes puestos de trabajo por los que han ido rotando. Les cuesta el cambio y la adaptación a un nuevo puesto hasta que aprenden las competencias exigidas.

Los responsables de los puestos de trabajo muestran una actitud positiva en orden a facilitar la inserción laboral de los alumnos con discapacidad. Se implican con el programa formativo ofertando espacios laborales de experimentación. Además explican los trabajos que deben realizar. A veces deben repetir las órdenes dadas con el fin de lograr mayor comprensión. Los responsables laborales entienden que colaborar en la inserción laboral de las personas con discapacidad intelectual es una dimensión ética que implica responsabilidad. Y de este modo contribuyen a propiciar la igualdad de oportunidades a sujetos que, de otro modo, se verían excluidos del mundo laboral. Con su apoyo favorecen la inserción de estos jóvenes en el mercado laboral ordinario.

Por último, y de cara a nuevas acciones de este tipo, convendría tener presentes los siguientes aspectos que implican mejora en la preparación, organización y proyección del periodo de prácticas:

1. Diseñar con precisión el puesto de trabajo junto con las competencias requeridas

2. Antes de enviarlos a realizar las prácticas se ha revelado como imprescindible:

- Conversar con los representantes de la empresa sobre las características diferenciales de este tipo de sujetos, principalmente sus comportamientos, actitudes, habilidades, destrezas y capacidades. 
- Explicar con claridad qué pueden hacer y hasta dónde pueden exigirles.

- Diseñar los procesos a seguir con este tipo de sujetos.

- Describir los niveles de exigencia en el trabajo y formalizar con ellos los pasos de cada tarea.

- Conversar sobre las relaciones que se pueden establecer, manteniendo un difícil equilibrio entre la exigencia y el estímulo.

- Es importante que observen las tareas durante un tiempo determinado con el fin de captar si su realización presenta problemas.

- Ante posibles problemas sociolaborales, buscar soluciones conjuntas.

Si se desea insertar socialmente a los alumnos con discapacidad, es imprescindible abrirles las puertas al ámbito laboral, puesto que la verdadera inserción solo se logra mediante el trabajo remunerado. La sociedad debe emplear recursos que propicien su inclusión y no regatear esfuerzos para que cada sujeto desempeñe el trabajo más acorde con sus capacidades. No se puede hablar de igualdad de oportunidades si no somos capaces de insertar a estos sujetos en el mercado de trabajo. Esta experiencia ha demostrado que con una formación adecuada y un plan de prácticas laborales diseñado con rigor y precisión, estos sujetos son capaces de desempeñar diferentes puestos de trabajo con resultados eficaces. En este sentido, Pérez Ferrando (2004: 361) indica que "es necesaria la creación de órganos o figuras intermedias, mediadoras que sirvan de nexo entre los diferentes espacios implicados". Sería conveniente que tras los resultados de esta investigación muchas empresas abrieran sus puertas a los sujetos con discapacidad, pues solo de este modo se podría hablar de igualdad de oportunidades.

\section{Referencias bibliográficas}

Charmaz, K. (2006): Construting Grounded Theory. A practical guide through qualitative analysis. Londres: Sage.

Glaser, B. G., y Strauss A. L. (1967): The Discovery of Grounded Theory: Strategies for Qualitative Research. Chicago: Aldine.

Miles, M. B., y Huberman, M. A. (1984): Qualitative data analysis. A sourcebook of new methods. Beverly Hills: Sage.

Murh, T. (1997): Atlas.ti- Visual Qualitative data analysis-management-Model Building-Release 4.1. Berlín: Short User's Manual.

Pallisera, M. (2001): "Integración laboral de personas con discapacidad. Aportaciones para la comprensión de estos procesos a partir de la investigación", en Bueno, J. J., Nuñez, T. e Iglesias, A.: Atención educativa a la diversidad en el nuevo milenio (XVIII Jornadas de Universidades y Educación Especial). A Coruña. Universidad de A Coruña, Servicio de Publicaciones. pp. 211-232.

Pérez Ferrando, M. V. (2004): "Análisis de la población juvenil que participa en curso de formación profesional ocupacional". Revista Interuniversitaria de Pedagogía Social, $n^{0}$ 11, $2^{\underline{a}}$ época, pp. 349-362.

Pérez Serrano, G. (2007): Investigación cualitativa. Métodos y técnicas. Madrid: La Muralla.

Pidgeon, N., y Henwood, K. (1997): "Grounded theory: practical implementación”, en John T. E. Richardson (Eds): Handbook of qualitative research methods for psychology and the social sciences. Leicester: BPS Books.

Ríos González, M. F. (2003): "El contenido de la formación permanente en las conferencias mundiales de la UNESCO". Revista Interuniversitaria de Pedagogía Social, $n^{\circ}$ 10, pp. 281-297.

Valls, M. J. (2005): “Escuchando la voz de las personas con discapacidad psíquica. Hacia una investigación cooperativa" Bordón, vol. 57, nº 3, pp. 337-349 


\section{Notas}

1 "Formación para el empleo e inserción laboral de jóvenes con discapacidad intelectual en el ámbito universitario (segunda fase)". Estudio con financiación pública Ref. CCGo6/UAM/HUM-=316).
DireCCión de la AUTORA: Martina Pérez Serrano. Universidad Autónoma de Madrid. Departamento de Didáctica y Teoría de la Educación. Facultad de Educación. 28049 Cantoblanco. Madrid. Correo electrónico: martina.perez@uam.es

Fecha de recepción del artículo: 08.XI.2007

Fecha de aceptación definitiva: 10.X.2007 


\section{MISCELÁNEA}

A criança em risco e a relação Escola-Família. Protecção e Sucesso Educativo

- PAUlo DELGADO

Hacia un espacio europeo de aprendizaje para toda la vida. Evolución y desarrollo de la Educación Permanente en la Unión Europea

- FERNANDO LÓPEZ NOGUERO

Escuela, educación y territorio. La organización en red local como estructura innovadora de atención a las necesidades socioeducativas de una comunidad

- JORDI LONGÁS, MIREIA CIVIS, JORDI RIERA, ANNABEL FONTANET, EDUARD LONGÁS, TOMÁS ANDRÉS . 
